\title{
Components of Diesel Exhaust Particles Diversely Enhance a Variety of Respiratory Diseases Related to Infection or Allergy: Extracted Organic Chemicals and the Residual Particles after Extraction Differently Affect Respiratory Diseases
}

\author{
Hirohisa Takano ${ }^{1,2 *}$, Rie Yanagisawa ${ }^{1}$, and Ken-ichiro Inoue ${ }^{1,2}$ \\ ${ }^{1}$ Environmental Health Sciences Division, National Institute for Environmental Studies, Tsukuba 305-8506, Japan \\ ${ }^{2}$ Inflammation and Immunology, Kyoto Prefectural University of Medicine, Kyoto 602-8566, Japan
}

Received 6 October, 2006; Accepted 24 October, 2006

\begin{abstract}
Summary Experimental and epidemiological studies have reported that diesel exhaust particles (DEP) can aggravate a variety of respiratory diseases including infection or allergy. However, the responsible components in DEP for the enhancement have not been identified. The present review demonstrates the different effects of the components of DEP on the respiratory diseases related to infection or allergy. We exposed mice to the organic chemicals (DEP-OC) and the residual carbonaceous nuclei (washed DEP) derived from DEP in the presence or absence of bacterial endotoxin (lipopolysaccharide: LPS) or allergen. In our first series of experiments, washed DEP combined with LPS synergistically exacerbated lung injury, which was concomitant with the enhanced lung expression of proinflammatory cytokines and chemokines, whereas DEP-OC combined with LPS did not. In contrast, our second series of experiments showed that DEP-OC, rather than washed DEP, enhanced allergen-related eosinophilic inflammation and proliferation of goblet cells in the airway epithelium, which was paralleled by the enhanced lung expression of eotaxin and interleukin5. However, washed DEP with ovalbmin showed less change and increased the lung expression of interferon- $\gamma$. It is suggested that DEP components diversely affect various types of respiratory diseases, while the combination of organic chemicals and carbonaceous nuclei (whole DEP) mostly aggravate respiratory diseases.
\end{abstract}

Key Words: diesel exhaust particle, bacterial endotoxin, allergen, cytokine, chemokine

\section{Introduction}

Previous epidemiological studies have shown that exposure to ambient particulate matters (PM) is associated with an increase in the morbidity and daily mortality caused by respiratory diseases $[1,2]$. Concentration of PM with a

\footnotetext{
*To whom correspondence should be addressed.

Tel \& Fax: +81-298-50-2334

E-mail: htakano@nies.go.jp
}

diameter less than $10 \mu \mathrm{m}$ (PM10) is related to daily hospital admissions caused by bronchial asthma, acute and chronic bronchitis, and by pulmonary infection. Recently, it has been demonstated that PM with a diameter less than $2.5 \mu \mathrm{m}$ (PM2.5) are more closely associated with the respiratory effects and the subsequent mortality than PM10 [3].

Diesel exhaust particles (DEP) are predominant constituents of PM2.5 in urban areas [4]. DEP can affect a variety of respiratory diseases. Our experimental studies have recently reported that DEP exacerbate allergic asthma $[5,6]$ and neutrophilic lung inflammation related to bacterial 
endotoxin from gram-negative bacteria [7].

DEP consist of carbonaceous nuclei which absorb a vast number of organic chemicals including polycyclic aromatic hydrocarbons [8]. DEP can be divided into the organic chemicals (DEP-OC) and the residual carbonaceous nuclei (washed DEP) derived from DEP. However, it is not clear which components in DEP are critical contributors to the enhancing effects or whether the identical component(s) in DEP is (are) associated with the aggravation of various respiratory diseases. In this article, we demonstrate that washed DEP, rather than DEP, exaggerate neutrophilic lung inflammation and the expression of proinflammatory molecules in the presence of bacterial endotoxin, while DEP-OC, rather than washed DEP, exaggerate eosinophilic lung inflammation with goblet cell proliferation, enhance the Th2 responses, and increase the production of antigen-specific $\mathrm{IgG1}$ in the presence of allergen.

\section{Carbonaceous Nuclei of DEP Predominantly Exag-} gerate Infection-Related Airway Inflammation [9, 10]

\section{Preparation of DEP-OC and washed DEP}

DEP were extracted with dichloromethane $\left(\mathrm{CH}_{2} \mathrm{Cl}_{2}\right)$ and sonicated for $5 \mathrm{~min}$. The suspension was centrifuged at $200 \mathrm{~g}$ for $20 \mathrm{~min}$. The supernatants were transferred to another tube and the residue was washed with dichloromethane. This manipulation was repeated for 3 times. The residual particles of DEP were prepared as washed DEP. The extracts were combined, evaporated, dissolved in $100 \%$ dimethyl sulfoxide (DMSO) and prepared as DEP-OC.

\section{Study Protocol}

In our first series of experiments, male ICR mice were divided into the following experimental groups. The vehicle group received phosphate-buffered saline (PBS) containing $0.025 \%$ Tween 80 and $0.25 \%$ DMSO. The washed DEP group or the DEP-OC group received $125 \mu \mathrm{g}$ of washed DEP or DEP-OC in the same vehicle, respectively. The lipopolysaccharide (LPS)group received $75 \mu \mathrm{g}$ of LPS ( $E$. coli B55: 05) dissolved in the vehicle. The washed DEP + LPS group or the DEP-OC + LPS group received combined administration of washed DEP or DEP-OC with LPS in the same vehicle, respectively. In each group, vehicle, DEP-OC, washed DEP, LPS, DEP-OC + LPS, or washed DEP + LPS was inoculated by the intratracheal route under anesthesia with $4 \%$ halothane.

\section{DEP-OC and washed DEP induce different lung histology}

The lung specimens stained with hematoxylin and eosin 24 hours after the intratracheal exposure showed that LPS induced moderate infiltration of neutrophils. The combined exposure to washed DEP and LPS markedly enhanced neutrophil sequestration, interstitial edema, and alveolar hemorrhage as compared with LPS exposed alone. The histological changes caused by DEP-OC + LPS exposure were less prominent than those by washed DEP + LPS exposure.

DEP-OC and washed DEP differently affect the expression of proinflammatory molecules

The protein concentrations of interleukin (IL)-1 $\beta$, macrophagr inflammatory protein (MIP)-1 $\alpha$, monocyte chemotactic protein (MCP)-1, and keratinocyte chemoattractant (KC) in the lung tissue supernatants 24 hours after the intratracheal exposure (Table 1,2) were below the detection limits in the washed DEP and the DEP-OC groups. LPS exposure significantly increased the protein concentrations of IL-1 $\beta$, MIP- $1 \alpha$, MCP-1, and KC as compared with vehicle exposure. The combined exposure to washed DEP and LPS resulted in further significant increases as compared with LPS exposed alone. The results on these proinflammatory molecules were concomitant with those on the neutrophilic inflammation with pulmonary edema. DEP-OC + LPS exposure did not increase the concentrations of these proinflammatory molecules as compared to LPS exposure.

The magnitude of mRNA expression in the lung was determined by semi-quantitative RT-PCR 4 hours after the intratracheal exposure. Exposure to LPS significantly increased the mRNA expression for IL-1 $\beta$ and MIP- $1 \alpha$ as compared to vehicle exposure. Exposure to washed DEP + LPS further increased the mRNA expression for these proinflammaotry molecules as compared to LPS exposure, whereas DEP-OC + LPS exposure did not. Moreover, LPS exposure increased the mRNA expression for Toll-like receptor (TLR) 2 as compared with the vehicle exposure. The expression of TLR2 was more prominent in the DEPOC + LPS and the washed DEP + LPS groups than in the LPS group. The expression was most prominent in the washed DEP + LPS group. Exposure to DEP-OC, washed DEP, LPS, or DEP-OC + LPS slightly increased the TLR4 expression as compared with vehicle exposure. A larger increase in the TLR4 expression was induced in the washed DEP + LPS group than in the LPS group (Fig. 1).

\section{Organic Chemicals in DEP Predominantly Exagger- ate Allergy-Related Airway Inflammation [11]}

\section{Study Protocol}

Male ICR mice were divided into 8 experimental groups. The vehicle group received PBS containing $0.025 \%$ Tween 80 and $0.25 \%$ DMSO. The washed DEP group or the DEPOC group received $50 \mu \mathrm{g}$ of washed DEP or DEP-OC in the same vehicle, respectively. The whole DEP group received $50 \mu \mathrm{g}$ of washed DEP and $50 \mu \mathrm{g}$ of DEP-OC mixed together in the vehicle. The OVA group received $1 \mu \mathrm{g}$ of ovalbumin (OVA: allergen) dissolved in the vehicle. The washed 
Table 1. Enhancement effects of washed DEP on the expression of proinflammatory molecules related to LPS

\begin{tabular}{lccccc}
\hline \multirow{2}{*}{ Group } & (n) & \multicolumn{4}{c}{ pg/lung supernatants } \\
\cline { 3 - 5 } & & IL-1 $\beta$ & MIP-1 $\alpha$ & MCP-1 & KC \\
\hline vehicle & 4 & $465 \pm 278$ & ND & ND & ND \\
washed DEP & 6 & ND & ND & ND & ND \\
LPS & 6 & $28561 \pm 10301^{*}$ & $2270 \pm 702$ & $4033 \pm 656$ & $3205 \pm 497^{*}$ \\
washed DEP + LPS & 6 & $47822 \pm 7883^{*, \dagger}$ & $4201 \pm 640^{\dagger}$ & $7477 \pm 811^{\dagger}$ & $4146 \pm 642^{*, \dagger \dagger}$ \\
\hline
\end{tabular}

We quantitated protein levels of interleukin-1 beta (IL-1 $\beta$ ), macrophage inflammatory protein-1 alpha (MIP-1 $\alpha$ ), macrophage chemoattractant protein-1 (MCP-1), and keratinocyte chemoattractant $(\mathrm{KC})$ in the lung tissue supernatants $24 \mathrm{~h}$ after the intratracheal treatments by ELISA. ${ }^{*} p<0.01$ vs vehicle group, ${ }^{\dagger} p<0.05$ vs LPS group, ${ }^{\dagger} p<0.01$ vs LPS group. Values are the mean \pm SEM of 5 animals in each group.

Table 2. Effects of DEP-OC on the expression of proinflammatory molecules related to LPS

\begin{tabular}{|c|c|c|c|c|c|}
\hline \multirow{2}{*}{ Group } & \multirow{2}{*}{ (n) } & \multicolumn{4}{|c|}{ pg/lung supernatants } \\
\hline & & IL-1 $\beta$ & MIP-1 $\alpha$ & MCP-1 & $\mathrm{KC}$ \\
\hline vehicle & 6 & $144 \pm 85.6$ & ND & ND & $58 \pm 58$ \\
\hline DEP-OC & 6 & $72.0 \pm 72.0$ & ND & ND & ND \\
\hline LPS & 6 & $44671 \pm 6481^{*}$ & $2989 \pm 433$ & $5286 \pm 992$ & $3903 \pm 597 *$ \\
\hline DEP-OC + LPS & 6 & $37176 \pm 7425^{*}$ & $1932 \pm 441^{\dagger}$ & $3712 \pm 602$ & $2417 \pm 536^{*}$ \\
\hline
\end{tabular}

We quantitated protein levels of IL-1 $\beta$, MIP-1 $\alpha$, and MCP-1, KC in the lung tissue supernatants 24 hours after the intratracheal treatments by ELISA. ${ }^{*} p<0.01$ vs vehicle group, ${ }^{\dagger} p<0.05$ vs LPS group. Values are the mean \pm SEM of 6 animals in each group.

(A)

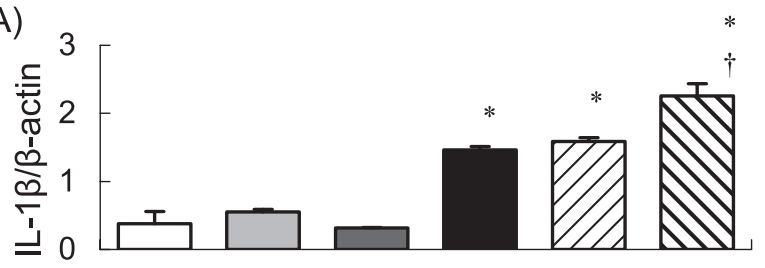

(B)

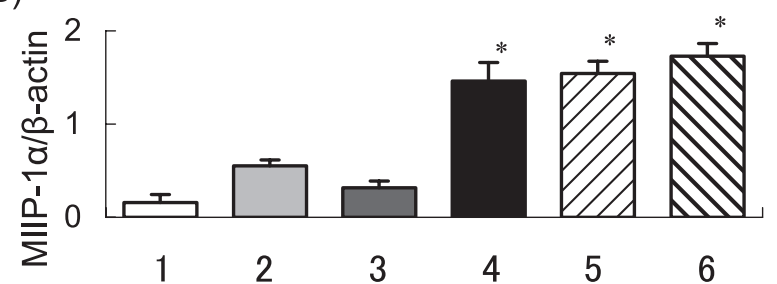

(C)



Fig. 1. Effects of DEP-OC and washed DEP on mRNA expression. We evaluated the magnitude of mRNA expression for IL-1 $\beta$ (A), MIP-1 $\alpha$ (B), Toll-like receptor 2 (TLR2) (C) and Toll-like receptor 4 (TLR4) (D) 4 hours after the intratracheal treatment by semi-quantitative RT-PCR. The amplification cycles were 33 and 35 for IL-1 $\beta$ (A), 30 and 32 for MIP-1 $\alpha$ (B), 30 and 32 for TLR2 (C), 28 and 30 for TLR4 (D). In each case, $\beta$-actin was amplified by the same protocol. For quantification, PCR bands in photographs of the gel were scanned by a densitometer linked to a computer analysis system. We used a published RT-PCR technique to measure relative differences in transcript levels after normalization against levels of the reference gene $\beta$-actin. The panel shows band density ratios of arbitrary density units for IL-1 $\beta$ (A), MIP-1 $\alpha$ (B), TLR2 (C) and TLR4 (D) to $\beta$-actin. The bottom number shows 1) vehicle group, 2) DEP-OC group, 3) washed DEP group, 4) LPS group, 5) DEP-OC + LPS group, 6) washed DEP + LPS group. ${ }^{*} p<0.01$ vs vehicle group; ${ }^{\dagger} p<0.05$ vs LPS group. Each ratio represents the mean \pm SEM of at least 3 animals per group. 
$\mathrm{DEP}+$ OVA group, the DEP-OC + OVA group, or the whole $\mathrm{DEP}+\mathrm{OVA}$ group received combined instillation of washed DEP, DEP-OC, or whole DEP together with OVA in the same vehicle, respectively. The all groups received OVA or vehicle every 2 weeks for 6 weeks, and DEP components or vehicle once a week for 6 weeks. In each group, vehicle, DEP-OC, washed DEP, whole DEP, OVA, DEP-OC + OVA, washed DEP + OVA, or whole DEP + OVA was inoculated by the intratracheal route under anesthesia with $4 \%$ halothane.

\section{DEP components induce different lung histology}

The lung specimens stained with H\&E or PAS $24 \mathrm{~h}$ after the last intratracheal exposure showed that infiltration of eosinophils and neutrophils (polymorphonuclear leukocytes: PMNs) and mononuclear cells and goblet cell proliferation were slight in the DEP-OC, the washed DEP, the whole DEP, and the OVA groups (Fig. 2). Exposure to OVA + DEP-OC or $\mathrm{OVA}+$ whole DEP induced more prominent infiltration of PMNs than that to OVA alone. The infiltration was most prominent on the exposure to OVA + whole DEP. Exposure to DEP components + OVA enhanced infiltration of the mononuclear cells around the airways as compared with vehicle exposure. Exposure to DEP-OC + OVA and whole DEP + OVA showed remarkable increases in the number of mononuclear cells as compared with OVA exposed alone.
Similar results were observed in the proliferation of goblet cells in the airway epithelium.

\section{DEP components differently affect Th1/Th2 balance}

The expression of Th1 and Th2 cytokines in the lung tissue supernatants $24 \mathrm{~h}$ after the final exposure demonstrated that combined intratracheal exposure to DEP-OC + OVA significantly increased the protein level of IL-5 as compared with OVA exposed alone (Table 3). The combined exposure to whole DEP + OVA resulted in a further significant increase in IL-5. Combined exposure to whole DEP + OVA resulted in a remarkable elevation of IL-13. Expression of IFN- $\gamma$, a Th1-type cytokine, was significantly greater in the washed DEP + OVA group than in the OVA group.

The protein level of eotaxin in the OVA group was significantly greater than in the vehicle group. The DEPOC + OVA group showed a further significant increase in eotaxin as compared with the OVA group (Table 4). Furthermore, the combined exposure to whole DEP + OVA significantly enhanced the expression of eotaxin. The expression of MIP-1 $\alpha$ was significantly greater in the whole DEP group than in the vehicle group. Exposure to whole DEP + OVA showed a further significant increase in MIP$1 \alpha$ as compared with exposure to vehicle, OVA, washed DEP + OVA, or DEP-OC + OVA.



Fig. 2. Histological changes in the murine lung $24 \mathrm{~h}$ after the last intratracheal instillation. The sections were stained with $\mathrm{H} \& \mathrm{E}$ to evaluate the degree of infiltration of polymorphonuclear cells (PMNs) or mononuclear cells around the all airways and with periodic acid Schiff (PAS) to evaluate the degree of proliferation of goblet cells in the bronchial epithelium. ${ }^{*} p<0.05$ vs vehicle group, ${ }^{* *} p<0.01$ vs vehicle group, ${ }^{\dagger} p<0.05$ vs OVA group, ${ }^{\dagger} p<0.01$ vs OVA group. Data are the means \pm SEM of at least 4 animals per group. 
Table 3. Effects of DEP components on the protein expression of Th1 and Th2 cytokines

\begin{tabular}{llccc}
\hline & & \multicolumn{3}{c}{$\mathrm{pg} /$ lung supernatants } \\
\cline { 3 - 5 } Group & $\mathrm{n}$ & $\mathrm{IFN}-\gamma$ & $\mathrm{IL}-5$ & $\mathrm{IL}-13$ \\
\hline vehicle & 8 & $561 \pm 37$ & $12.3 \pm 0.3$ & $1.29 \pm 1.29$ \\
DEP-OC & 8 & $584 \pm 56$ & $13.2 \pm 0.4$ & $3.32 \pm 2.00$ \\
washed DEP & 8 & $558 \pm 25$ & $12.8 \pm 0.3$ & $1.13 \pm 0.43$ \\
whole DEP & 8 & $534 \pm 25$ & $31.8 \pm 16.7$ & $29.24 \pm 13.3$ \\
OVA & 8 & $536 \pm 60$ & $33.5 \pm 13.0$ & $22.31 \pm 9.57$ \\
DEP-OC + OVA & 8 & $621 \pm 49$ & $75.3 \pm 18.0^{*, \dagger}$ & $39.42 \pm 27.4$ \\
washed DEP + OVA & 8 & $696 \pm 74^{\dagger}$ & $22.9 \pm 7.5$ & $6.27 \pm 5.41$ \\
whole DEP + OVA & 8 & $455 \pm 75^{\S}$ & $184.2 \pm 23.1^{\S}$ & $165.03 \pm 36.3^{\S}$ \\
\hline
\end{tabular}

8 groups of mice were intratracheally inoculated with vehicle, DEP-OC, washed DEP, whole DEP, OVA, or the combination of DEP components and OVA for 6 weeks. Lungs were removed $24 \mathrm{~h}$ after the last intratracheal administration. Protein levels of cytokines in the lung tissue supernatants were analyzed using ELISA. ${ }^{*} p<0.01$ vs vehicle group, ${ }^{*} p<0.05$ vs. OVA group, ${ }^{\$} p<0.01$ vs washed DEP + OVA group, ${ }^{\S} p<0.01$ vs other groups. Data are the means \pm SEM of 8 animals per group.

Table 4. Effects of DEP components on the protein expression of chemokines

\begin{tabular}{|c|c|c|c|}
\hline \multirow{2}{*}{ Group } & \multirow{2}{*}{$\mathrm{n}$} & \multicolumn{2}{|c|}{$\mathrm{pg} /$ lung supernatants } \\
\hline & & Eotaxin & MIP-1 $\alpha$ \\
\hline vehicle & 8 & $36.1 \pm 17.6$ & $2.17 \pm 1.13$ \\
\hline DEP-OC & 8 & $116.1 \pm 4.94$ & $5.16 \pm 1.56$ \\
\hline washed DEP & 8 & $130.9 \pm 6.82$ & $24.71 \pm 7.58$ \\
\hline whole DEP & 8 & $255.4 \pm 108$ & $53.79 \pm 7.28 * *$ \\
\hline OVA & 8 & $355.3 \pm 146^{*}$ & $15.12 \pm 2.01$ \\
\hline DEP-OC + OVA & 8 & $765.1 \pm 164 * *, \uparrow$ & $10.88 \pm 10.1$ \\
\hline washed DEP + OVA & 8 & $222.8 \pm 75.5$ & $12.09 \pm 1.73$ \\
\hline whole DEP + OVA & 8 & $1375.9 \pm 169^{\S}$ & $76.45 \pm 22.8^{* *, 9, \dagger \dagger}$ \\
\hline
\end{tabular}

8 groups of mice were intratracheally inoculated with vehicle, DEP-OC, washed DEP, whole DEP, OVA, or the combination of DEP components and OVA for 6 weeks. Lungs were removed $24 \mathrm{~h}$ after the last intratracheal administration. Protein levels of chemokines in lung tissue supernatants were analyzed using ELISA. ${ }^{*} p<0.05$ vs vehicle group, ${ }^{*} p<0.01$ vs vehicle group, ${ }^{\dagger} p<0.05$ vs OVA group, ${ }^{\dagger} p<0.01$ vs OVA group, ${ }^{\top} p<0.01$ vs washed DEP + OVA and DEP-OC + OVA group, ${ }^{\circledR} p<0.01$ vs other groups. Data are the means \pm SEM of 8 animals per group.

DEP-OC and whole DEP enhance OVA-specific IgGI

Exposure to DEP-OC significantly increased the production of OVA-specific IgG1 as compared with vehicle exposed alone (Fig. 3). Combined exposure to whole DEP + OVA markedly enhanced OVA-specific IgG1.

\section{Conclusion}

Our first series of experiments has demonstrated that washed DEP synergistically exacerbate neutrophilic lung inflammation with edema and alveolar hemorrhage in the presence of LPS, which is concomitant with the expression of IL-1 $\beta$, MIP-1 $\alpha$, MCP-1, and KC. The mRNA expression for TLR2 and TLR4 in the lung is increased by the combined exposure to washed DEP + LPS.

The second series of our experiments has shown that intratracheal exposure to DEP-OC, rather than washed DEP, enhances eosinophilic inflammation and the proliferation of goblet cells in the airway epithelium in allergen-stimulated mice, which is paralleled by the expression of proinflammatory molecules such as eotaxin and IL-5 in the lung as well as the production of antigen-specific IgG1. In contrast, washed 


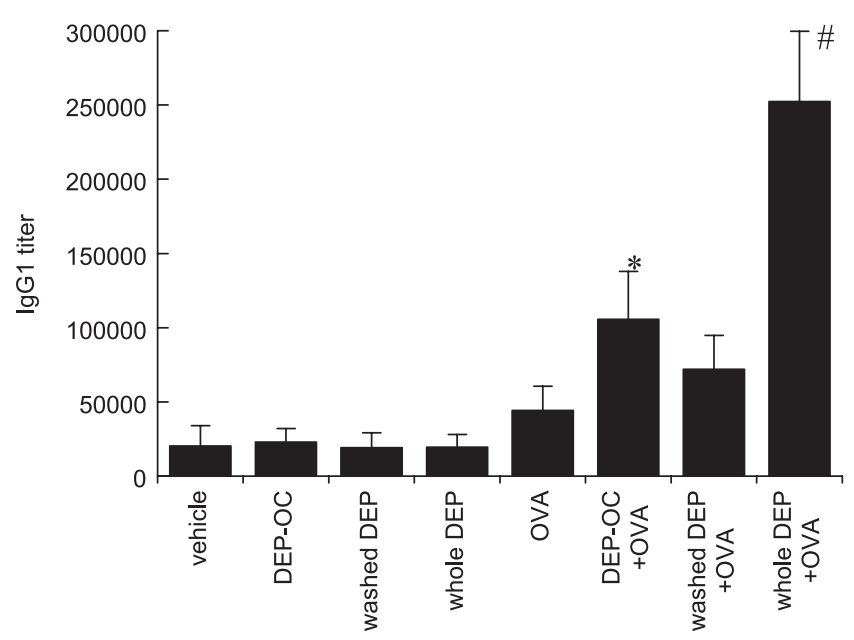

Fig. 3. To evaluate adjuvant activity of DEP components on antigen induced Ig production, we measured OVA-specific IgG1 in the serum $24 \mathrm{~h}$ after the last intratracheal instillation using ELISA. ${ }^{*} p<0.05$ vs vehicle group, ${ }^{*} p<0.01$ vs other groups. Data are the means \pm SEM of 8 animals per group.



Fig. 4. DEP components differently affect a variety of respiratory inflammation. The residual carbonaceous nuclei of DEP can predominantly enhance Th1 response and possibly neutrophilic inflammation, while the organic chemicals in DEP primarily accelerate Th2 response and possibly eosinophilic inflammation. In both models of respiratory inflammation, however, whole DEP most prominently affect the aggravation as compared with each component of DEP.

DEP with allergen increases the expression of IFN- $\gamma$. All the enhancing effects are mostly remarkable in the whole DEP + OVA group than in the other groups.

These studies suggest that the residual carbonaceous nuclei of DEP predominantly contribute to the aggravation of lung injury related to bacterial toxin rather than the organic chemicals in DEP, while whole DEP most syner- gistically exacerbate it [7]. The enhancing effects may be mediated through the expression of proinflammatory molecules, including cytokines, chemokines, and TLRs. In contrast, the organic chemicals in DEP, rather than the residual carbonaceous nuclei of DEP, exaggerate allergic airway inflammation with goblet cell proliferation, enhance the Th2 responses, and increase the production of antigen-specific IgG1, whereas whole DEP cause the most remarkable aggravation. Taken together, DEP components differently affect a variety of respiratory diseases. It is possible that the residual carbonaceous nuclei of DEP predominantly enhance Th1 response, while the organic chemicals in DEP primarily accelerate Th2 response (Fig. 4). In both models, however, whole DEP most prominently affect the aggravation of the respiratory diseases as compared with each component of DEP.

\section{Acknowledgments}

The authors wish to acknowledge Dr. Takahiro Kobayashi for preparation of DEP extraction, National Institute for Environmental Studies. We also thank Miho Sakurai and Naoko Ueki for technical assistance.

\section{References}

[1] Dockery, D.W., Pope, C.A. III, Xu, X., Spengler, J.D., Ware, J.H., Fay, M.E., Ferris, B.G., Jr., and Speizer, F.E.: An association between air pollution and mortality in six U.S. cities. N. Engl. J. Med., 329, 1753-1759, 1993.

[2] Schwartz, J.: Air pollution and daily mortality: a review and meta analysis. Environ. Res., 64, 36-52, 1994.

[3] Peters, A., Wichmann, H.E., Tuch, T., Heinrich, J., and Heyder, J.: Respiratory effects are associated with the number of ultrafine particles. Am. J. Respir. Crit. Care Med., 155, 1376-1383, 1997.

[4] Yoshizumi, K., Watanabe, T., and Ishii, K.: The source of apportionment evolution of ambient particle matter in Tokyo Metropolitan area by a receptor oriental model. Annual report of the Tokyo metropolitan research institute for environmental protection, pp. 3-10, 1989.

[5] Ichinose, T., Takano, H., Miyabara, Y., Yanagisawa, R., and Sagai, M.: Murine strain differences in allergic airway inflammation and immunoglobulin production by a combination of antigen and diesel exhaust particles. Toxicology, 122, 183-192, 1997.

[6] Takano, H., Yoshikawa, T., Ichinose, T., Miyabara, Y., Imaoka, K., and Sagai, M.: Diesel exhaust particles enhance antigeninduced airway inflammation and local cytokine expression in mice. Am. J. Respir. Crit. Care Med., 156, 36-42, 1997.

[7] Takano, H., Yanagisawa, R., Ichinose, T., Sadakane, K., Yoshino, S., Yoshikawa, T., and Morita, M.: Diesel exhaust particles enhance lung injury related to bacterial endotoxin through expression of proinflammatory cytokines, chemokines, and intercellular adhesion molecule-1. Am. J. Respir. 
Crit. Care Med., 165, 1329-1335, 2002.

[8] International Agency for Research on Cancer: Research on Cancer. IARC Monographs on the evaluation of Carcinogenic Risks to Humans: Diesel and Gasoline Exhaust and Some Nitroarenes. Lyon, France, 46, pp. 1-240, 1989.

[9] Yanagisawa, R., Takano, H., Inoue, K., Ichinose, T., Sadakane, K., Yoshino, S., Yamaki, K., Kumagai, Y., Uchiyama, K., Yoshikawa, T., and Morita, M.: Enhancement of acute lung injury related to bacterial endotoxin by components of diesel exhaust particles. Thorax, 58, 605-612, 2003.
[10] Arimoto, T., Inoue, K., Yanagisawa, R., and Takano, H.: Diesel exhaust particles synergistically enhance lung injury and oxidative stress induced by bacterial endotoxin. J. Clin. Biochem. Nutr., 38, 133-137, 2006.

[11] Yanagisawa, R., Takano, H., Inoue, K., Sakurai, M., Ichinose, T., Sadakane, K., Yoshino, S., Yamaki, K., Yoshikawa, T., and Hayakawa, K.: Components of diesel exhaust particles differentially affect Th1/Th2 response in a murine model of allergic airway inflammation. Clin. Exp. Allergy, 36, 386395, 2006. 